\title{
Sensitivity to amphotericin B of a Naegleria sp. isolated from a case of primary amoebic meningoencephalitis
}

\author{
RODNEY F. CARTER
}

From the Department of Pathology, The Adelaide Children's Hospital Inc., North Adelaide, South Australia

SYNOPSIS An amoeba of the genus Naegleria causing fatal meningoencephalitis in a human subject ${ }_{-}$ has been investigated for its sensitivity to several drugs. Penicillin, sulphadiazine, chloramphenicol, oxytetracycline hydrochloride, streptomycin, methotrexate, emetine, quinine, and metronidazole had no effect on the organism in vitro at levels in excess of those likely to be attained therapeutically in the brain. Amphotericin B was highly amoebicidal in vitro and protected mice from infection with the organism. Used in high dosage by the intraventricular as well as the intravenous route,,$\frac{\subseteq}{<}$ this drug might be successful in the treatment of further cases of primary amoebic meningoence- $-\overrightarrow{0}$ phalitis.

In 1965 Fowler and Carter demonstrated that freeliving amoebae can invade the human brain by the olfactory route to cause a rapidly fatal meningitis very like the fulminating bacterial variety. Thirty-four cases of this disease, which has come to be known as primary amoebic meningoencephalitis, have now been reported (Patras and Andujar, 1966; Butt, 1966; Callicott, 1968; Červa and Novak, 1968; Carter, 1968); the causative organisms have been isolated from two patients and identified as amoebae of the genus Naegleria (Butt, Baro, and Knorr, 1968; Culbertson, Ensminger, and Overton, 1968; Carter, 1968). A recent paper (Carter, 1968) provides details concerning the early diagnosis of the disease but until now lack of knowledge of the drug sensitivity of Naegleria has stood in the way of effective treatment.

Reported here is an investigation into the effect of several well-known drugs on the Naegleria sp. isolated from the case in South Australia (Carter, 1968).

\section{EXPERIMENTAL DETAILS}

SOURCE AND PREPARATION OF AMOEBAE Originally isolated from a human brain at necropsy, the amoebae have been maintained by fortnightly animal passage. Female Swiss white mice weighing 10 to $15 \mathrm{~g}$, fed ad libitum on standard pellet food and housed at $21^{\circ} \mathrm{C}$, are anaesthe-

Received for publication 5 December 1968. tized to falling point with diethyl ether and inoculated intranasally with an amoebic suspension. Moribund in five to six days with amoebic meningoencephalitis, they $\mathbb{D}$ are killed with diethyl ether and their brains removed aseptically into 2 to $5 \mathrm{ml}$ of sterile, chemically definedo proteose-peptone-glucose medium (PPG) (Band, 1959) in a $20 \mathrm{ml}$ McCartney bottle. Vigorous agitation produces PPG suspensions of amoebae which range in concentration from 1,000 to 8,000 per $\mathrm{cmm}$ and remain viable and infective for up to four weeks. For routine passage, further mice are inoculated after nine days with $20 \mathrm{cmm}$ of PPG suspension containing approximately $2,000-$ amoebae per $\mathrm{cmm}$.

Water suspensions of amoebae, of similar concentration, viability, and infectivity, are prepared by taking the brains into sterile water instead of PPG; the preparations are shaken vigorously three times in one hour and theo suspensions then decanted from the brains.

DRUGS Throughout this paper, doses and levels refer $N$ to drugs as base, not the salt used; the water referred tor is sterile 'water for injection, BP'. Drugs tested are listed in the table; sterile solutions in water were freshly prepared, either under aseptic conditions using sterile stock or by membrane filtration (pore size $0.45 \mu$ ), and used within two hours. Immobilization and cultural inhibition ${ }^{+}$ tests were set up using sterile doubling dilutions of the drugs in water so designed that the final range of con-웅 centrations encompassed the levels of the drugs therapeutically attainable in human cerebrospinal fluido (see Table).

Amphotericin B was in the 'solubilized' form, con $\bar{x}$ taining sodium desoxycholate and buffers as suspending 
TABLE

DRUG SENSITIVITY IN VITRO OF Naegleria Sp. COMPARED WITH DRUG LEVELS ATTAINABLE IN HUMAN CEREBROSPINAL FLUID

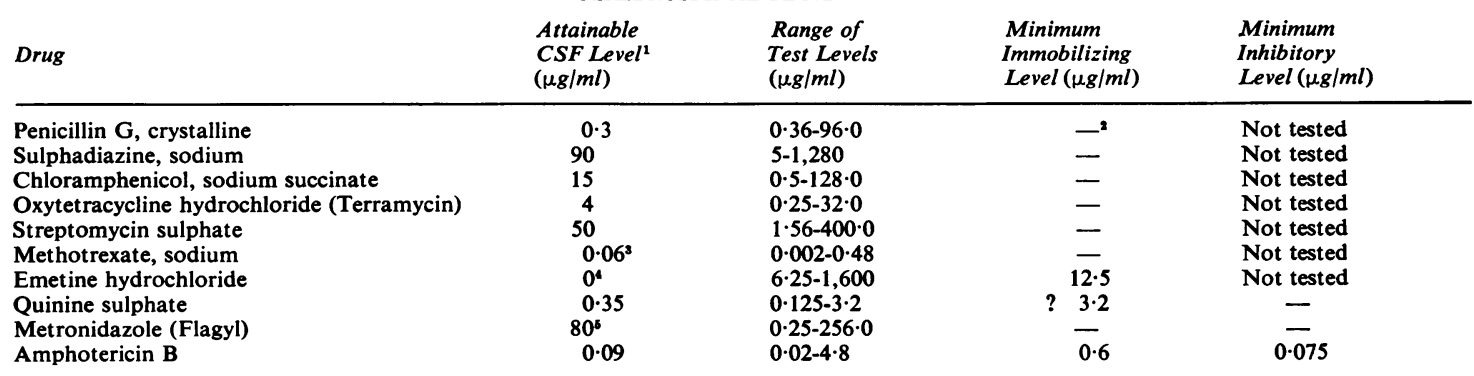

1 Based on Goodman and Gilman (1965) except where indicated.

-Drug ineffective at levels tested

Cramblett (1959).

- Parmer and Cottrill (1949).

- Davies (1967).

agents. Since it is possible that the amoebae, which are bile-soluble, might be affected by the amphotericinsuspending agents, solutions containing amounts of these agents equal to those in the test dilutions of amphotericin B were used as controls throughout.

For the animal protection trials, amphotericin B and the amphotericin-suspending agent control solution were prepared as above. Mucilage of tragacanth BP, diluted 1 volume to 3 volumes of water, was used to suspend metronidazole and alone to act as the control solution. Drug and control solutions were made up freshly every three days and stored at 0 to $4^{\circ} \mathrm{C}$.

IMMOBILIZATION TESTS Equal portions of five to sevenday-old water suspensions of amoebae ( $p \mathrm{H}$ 7.9) and drug dilutions were thoroughly mixed and mounted as hanging drops, using ordinary coverslips and microslides sealed with petroleum jelly. Controls were prepared in the same way using water instead of the drug dilutions; in the case of amphotericin B additional controls were prepared using the amphotericin-suspending agents control solution. In all tests a hanging drop of the plain amoebic suspension was also prepared to act as a further control. The initial concentration of the amoebic suspension was adjusted so that the drops, about $5 \mathrm{~mm}$ in diameter and $15 \mathrm{cmm}$ in volume, contained 500 to 1,000 amoebae. The preparations were left inverted for five minutes to allow settling and adherence of amoebae to the coverslips, and then kept in the dark at $21^{\circ} \mathrm{C}$; maintenance at $37^{\circ} \mathrm{C}$ was impracticable owing to the rapid transformation of the amoebae to cystic forms at this temperature. Microscopical examination at a quarter, two, six, 24 , and 48 hours included rough counts per high-power field to detect any marked change in numbers of amoebae.

In all control preparations amoebae showed no change in numbers and remained motile and adherent to the undersurfaces of coverslips throughout the test period. Immobilization was judged to have occurred only when all the amoebae in a preparation had either disinte- grated, or showed rounding, shrinkage, loss of adherence to the coverslip, and cessation of all cytoplasmic granular movement.

CULTURAL INHIBITION An agar doubling dilution technique was used, drugs being incorporated into $1.25 \%$ agar (Oxoid no. 3) plates by mixing equal portions of drug dilution and $2.5 \%$ agar at $48^{\circ} \mathrm{C}$ before pouring; exposure of drugs to $48^{\circ} \mathrm{C}$ did not exceed one hour. Controls were prepared using water instead of drug dilution, with the addition in the case of amphotericin $B$ of an equivalent range of dilutions of the amphotericinsuspending agents control solution. Two drops $(40 \mathrm{cmm})$ of a suspension of bacteria (necessary as food for the amoebae) were then spread over a $4 \mathrm{~cm}$ diameter area of the plates, which were allowed to dry at $37^{\circ} \mathrm{C}$ for two hours. To make the bacterial suspension, Escherichia coli cultured on nutrient agar at $37^{\circ} \mathrm{C}$ for 16 hours were suspended in water at an approximate concentration of $5 \times 10^{8} / \mathrm{ml}$ ( 1 slope culture in $2.5 \mathrm{ml}$ water). All plates (final $p \mathrm{H}$ about 6.0 ) were then inoculated centrally with $20 \mathrm{cmm}$ of amoebic suspension in proteose-peptoneglucose medium adjusted to contain 25 to 50 amoebae per $\mathrm{cmm}$, sealed with Sellotape, incubated in darkness at $37^{\circ} \mathrm{C}$, and examined microscopically at one, two, and six days.

For the first two days the amoebae in all control cultures proliferated rapidly, doubling their numbers about every four hours and migrating peripherally as an expanding ring of densely packed organisms; at six days the nutrient bacteria had been consumed and many amoebae had either encysted or disintegrated. Cultural inhibition was judged to have occurred if at the end of 48 hours the amoebae in a plate either showed no proliferation at all or had disappeared. The possibility that amphotericin B might inhibit the amoebae by interfering with the nutrient bacteria is unlikely since the bacteria were shown to support normal amoebic growth after subculture from six-day-old plates containing the highest concentration of the drug. 
ANimal Protection Mice, $15 \mathrm{~g}$, were used, being inoculated, fed, and housed as for routine passage. For the amphotericin B trial, 20 mice received a daily intraperitoneal injection of $0.25 \mathrm{ml}$ solution containing $0.112 \mathrm{mg}$ of drug $(7.5 \mathrm{mg} / \mathrm{kg}$ body weight), and 20 the same amount of an equivalent dilution of the amphotericin-suspending agents control solution; injections continued for 14 days. For the metronidazole trial, 10 mice were each given by dosing needle a daily oral dose of $0.25 \mathrm{ml}$ of suspension containing $15 \mathrm{mg}$ of drug ( $1 \mathrm{~g} / \mathrm{kg}$ body weight), and 10 mice the same amount of control solution; dosing continued for 10 days.

All animals were inoculated intranasally with 40,000 amoebae $\left(133 \times \mathrm{LD}_{50}\right) 24$ hours after the first dose of drug. Survivors were reinoculated in the same way after 77 days, their subsequent death with the typical amoebic disease discounting the possibility that their initial survival might have been due to an innate resistance to the infection. Histological examination confirmed that all animals dying in the trials were suffering from severe amoebic meningoencephalitis.

Results were analysed statistically using the chi square test.

BIOASSAY OF AMPHOTERICIN IN MOUSE SERUM Test serum was obtained from six uninoculated $15 \mathrm{~g}$ mice injected with amphotericin B as above for 14 days and exsanguinated by cardiac puncture three hours after the last injection. Control serum from untreated stock mice was obtained in the same way. The test serum was assayed for amphotericin B activity by the cultural inhibition technique of Taylor, Lynch, Taylor, and Weiser (1958) using as the test organism an unspecified strain of Candida albicans inhibited by a minimum amphotericin B concentration of $0.3 \mu \mathrm{g} / \mathrm{ml}$.

The result was checked by a second assay using

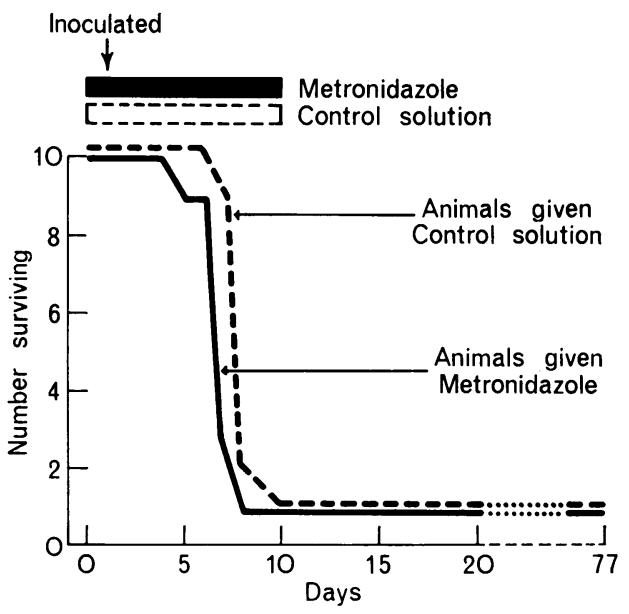

FIG. 1 Failure of metronidazole to protect mice from Naeglerial meningoencephalitis. immobilization of the Naegleria sp. as an indicator of $\frac{\stackrel{0}{7}}{\overrightarrow{2}}$ drug activity. Control serum was mixed with an equal 으․ amount of drug solution of known concentration, and $\overrightarrow{\vec{F}}$ test serum was mixed with an equal amount of ampho- $\stackrel{\vec{P}}{+}$ tericin-suspending agents solution of equivalent con- $\bar{C}$ centration. Both were then serially diluted in water, $\overline{\bar{\sigma}}$ mixed with amoebae, and prepared and examined as $\overline{\bar{S}}$ described for the immobilization tests. Concentration of $\mathbb{\Phi}$ drug in the test serum was calculated by comparing the maximum dilutions of test and control sera at which $\infty$ immobilization occurred in 48 hours. A preparation of $\vec{\circ}$ control serum mixed with amphotericin-suspending agents solution was also included in the assay to eliminate $\vec{\omega}$ the possibility that either normal mouse serum or the amphotericin-suspending agents solution might interfere용 with the results by causing immobilization at the dilutions used.

By both methods the level of amphotericin B in the test serum was estimated to be $1 \cdot 2 \mu \mathrm{g} / \mathrm{ml}$.

\section{RESULTS AND DISCUSSION}

Results of immobilization and cultural inhibition tests are given in the table. Results of animal protection trails are depicted in Figures 1 and 2.

Penicillin, sulphadiazine, chloramphenicol, oxy-৫ tetracycline hydrochloride, and streptomycin showed no immobilizing effect at levels far in excess of those attainable in human cerebrospinal fluid. These drugs have been reported as ineffective in treating many human cases of the disease, and $\stackrel{\square}{\varrho}$ accordingly they were not investigated further.

Methotrexate was selected as a representative of the folic-acid-inhibiting drugs because of its effectiveness as a cytotoxic agent when given intrathecally ino

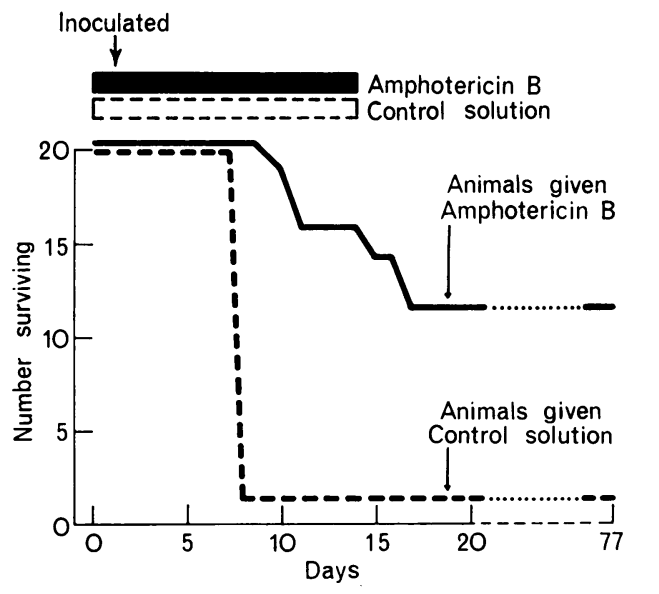

FIG. 2 Protection of mice from Naeglerial meningoencephalitis by amphotericin $B$. 
cerebral leukaemia (Cramblett, 1959). However, levels much higher than those usually maintained in cerebrospinal fluid did not produce any immobilizing effect, so further studies with this drug were not considered worthwhile.

The minimum immobilizing concentration of emetine hydrochloride was found to be much the same for the Naegleria sp. as it is for E. histolytica (Balamuth, 1953). This drug, however, has apparently been of little or no use in the treatment of cerebral infections due to $E$. histolytica: only two cases of cure have been reported (Collard and Kendall, 1947; Lenshoek, Baumann, and Wielenga, 1958) and even these are doubtful since in neither case was the diagnosis confirmed by direct observation or culture of amoebae from the central nervous system. The negligible levels of emetine reached in animal brains (Parmer and Cottrill, 1949) suggest that the drug would be of no value in treating cerebral infections. Certainly it had no effect in one human case of the Naeglerial disease where it was used for three days before death (Carter, 1968); its further investigation appeared unprofitable and was therefore abandoned.

Quinine was tested because of its action against the protozoa of malaria and its ability to pass the blood-brain barrier. Incomplete immobilization occurred only at a drug level greater than that likely to be tolerated by the brain; cultural inhibition was not observed.

Metronidazole, well known in the treatment of trichomoniasis, has recently been shown to be effective in systemic infections with E. histolytica (Powell, Macleod, Wilmot, and Elsdon-Dew, 1966) and to reach high levels in human cerebrospinal fluid (Davies, 1967); it was therefore fully investigated. Failure of the drug either to immobilize or inhibit the Naegleria sp. or to protect animals from infection at doses equivalent weight for weight to 18 times the maximum human dose (Fig. 1) suggests that metronidazole is unlikely to be effective in treating the human disease.

Amphotericin B was included in the study because of its established value in treating fungal infections of the central nervous system (Andriole and Kravetz, 1962) and its demonstrated effect, both in vitro (McMillan, 1960) and clinically (Prata, 1963) against protozoa of the genus Leishmania. The drug was found to be rapidly amoebicidal in vitro, disintegration of the amoebae in the immobilization test being complete at high drug levels within 15 minutes and advanced at the minimum immobilizing level of $0.6 \mu \mathrm{g} / \mathrm{ml}$ in two hours, very little change occurring thereafter. The cultural inhibition test produced a similar result, amoebae either proliferating normally or completely disappearing from the plates within 24 hours, but here the drug was effective at a much lower concentration; two separate tests with different batches of reagents and amoebae each showed a minimum inhibitory level of $0.075 \mu \mathrm{g} / \mathrm{ml}$. By comparison with the $0.2 \mu \mathrm{g} / \mathrm{ml}$ required to inhibit Cryptococcus neoformans (Gold, Stout, Pagano, and Donovick, 1956), this level indicates a high degree of sensitivity of Naegleria sp. to amphotericin B. Moreover, it can be seen from the Table to be lower than the level attainable by intravenous therapy in human cerebrospinal fluid. The animal protection trial clearly demonstrated a drug effect in vivo, the results (Fig. 2) being highly significant with respect both to the marked protection of drugtreated animals during the time the drug was given $(\mathrm{P}=<0.001$ at 14 days) and the overall survival at 77 days of $60 \%$ of drug-treated animals compared with $5 \%$ of controls $(\mathrm{P}=>0.001<0.01)$. The drug dosage in the trial $(7.5 \mathrm{mg} / \mathrm{kg})$, although lower than that required to protect mice from many fungal infections (Sternberg, Wright, and Oura, 1956), appears to be greater than that which human subjects can tolerate. However, if one considers the high rate of drug metabolism in small animals and uses the formula of Bushby (1963), based on surface areas rather than weights, to adjust the dose of amphotericin B from man to mouse, the dosage in the trial can be shown to be comparable with that customarily used in man. The bioassay of mouse serum supports this conclusion, the level of drug found $(1 \cdot 2 \mu \mathrm{g} / \mathrm{ml})$ being much the same as that in the serum of human subjects receiving the drug intravenously in the usual dosage (Goodman and Gilman, 1965 , p. 1295).

These experimental findings suggest that amphotericin B is likely to be effective against Naegleria sp. in the human brain. Whether or not the drug can be made to reach effective levels in the brain in time to arrest and cure this fulminating disease is another matter. Intravenous administration alone would probably be unsuccessful; the drug is usually given by this route as an initial very small dose which is only slowly increased to the maximum to avoid possible toxic effects. Even the most rapid schedule recommended (Goodman and Gilman, 1965) is unlikely to produce effective levels in the cerebrospinal fluid in less than four days, by which time most patients would have died. Intrathecal administration into the lumbar sac is more promising since the maximum dose can be given on the first occasion (Andriole and Kravetz, 1962) and drug concentration in the cerebrospinal fluid should be higher than that attained by intravenous therapy. However, for the drug to be rapidly distributed it should be mixed before injection with a large volume of cerebrospinal fluid (Rieselbach, Di Chiro, Freireich, and Rall, 1962), the withdrawal of which 
is a potentially dangerous manoeuvre in patients with the brain swelling often found in the present disease. The intracisternal route (Winn, 1963) would ensure more rapid drug distribution but is likely to be at least as dangerous. Intraventricular administration, preferably via an Ommaya reservoir and catheter (Witorsch, Williams, Ommaya, and Utz, 1965), is probably the best method to use since it avoids the hazards of intrathecal injection and would ensure very rapid distribution of the drug throughout the ventricles and subarachnoid space (Di Chiro, 1966). The dosage suggested by Witorsch et al in the treatment of cryptococcal meningitis is $0.1 \mathrm{mg}$ initially, increasing slowly to a maximum of 0.5 to $1.0 \mathrm{mg}$ three times a week. In the present disease it is probably justifiable to risk neurological complications and give 0.5 to $1.0 \mathrm{mg}$ initially, and daily for the first few days. Such dosage has been suggested by Kress and Cantrell (1963) following their experience with a patient who survived, with only minor neurological sequelae, a single intraventricular dose of $5.0 \mathrm{mg}$. Most of the amoebae in this disease are situated in the meninges and superficial cortex (Carter, 1968) and should be readily accessible to amphotericin B given intraventricularly. However, some may be present in areas that can best be reached by blood-borne drug, and it is suggested that intravenous treatment be carried out concurrently.

I must emphasize the need for prompt treatment in the maximum dosage permissible, by whatever route the drug is given, if it is to act in time to arrest the disease. The optimum duration of treatment, if initially successful, cannot be predicted. All observations on the disease so far indicate that it is an acute process, and cystic organisms or abscesses have not been found in the brain. However, the induction of a more chronic or relapsing form by treatment is a possibility always to be kept in mind.

I am grateful for the encouragement and advice given by Dr Malcolm Fowler, Director of Pathology, Adelaide Children's Hospital, and Professors J. S. Robertson and D. Rowley, Departments of Pathology and Microbiology, University of Adelaide.

Drugs and media were prepared by the Pharmacy and Bacteriology Departments of the Adelaide Children's
Hospital; May and Baker (Australia) Pty Ltd. provided the metronidazole. Mr A. McNeil and staff of the Central Animal House, University of Adelaide, supplied $\overrightarrow{\vec{\omega}}$ the mice. The work was assisted by a grant from the Research Trust of the Adelaide Children's Hospital Inc.

\section{REFERENCES}

Andriole, V. T., and Kravetz, H. M. (1962). J. Amer. med. Ass., $180,269$.

Balamuth, W. (1953). Amer. J. trop. Med. Hyg., 2, 191.

Band, R. N. (1959). J. gen. Microbiol., 21, 80.

Bushby, S. R. M. (1963). In Experimental Chemotherapy, edited byR. J. Schnitzer and F. Hawking, vol. 1, p. 50. Academic Press, $\vec{\omega}$ New York.

Butt, C. G. (1966). New Engl. J. Med., 274, 1473.

- Baro, C., and Knorr, R. W. (1968). Amer. J. clin. Path. 49, 256 (abstr).

Callicott, J. H., Jr (1968). Ibid, 49, 84.

Carter, R. F. (1968). J. Path. Bact., 96, 1.

Cerva, L., and Novăk, K. (1968). Science, 160, 92

Collard, P., and Kendall, D. (1947). Lancet, 2, 17.

Cramblett, H. G. (1959). Amer. J. Dis. Child., 97, 805.

Culbertson, C. G., Ensminger, P. W., and Overton, W. M. (1968) J. Protozool., 15, 353.

Davies, A. H. (1967). Brit. J. vener. Dis., 43, 197.

Di Chiro, G. (1966). Acta radiol. (Diagn.) (Stockh.), 5, 988.

Fowler, M., and Carter, R. F. (1965). Brit. med. J., 2, 740.

Gold, W., Stout, H. A., Pagano, J. F., and Donovick, R. (1956) $\rightarrow$ Antibiotics Ann., 1955-56, 579, New York.

Goodman, L. S., and Gilman, A. (1965). The Pharmacological Basis of. Therapeutics, 3rd ed. Macmillan, New York.

Kress, M. B., and Cantrell, J. R. (1963). Arch. intern. Med., 112, 386.

Lenshoek, C. H., Baumann, C., and Wielenga, D. K. (1958). Arch. chir. neerl., 10, 34.

McMillan, B. (1960). Ann. trop. Med. Parasit., 54, 293.

Parmer, L. G., and Cottrill, C. W. (1949). J. Lab. clin. Med., 34, 818

Patras, D., and Andujar, J. J. (1966). Amer. J. clin. Path., 46, 226.

Powell, S. J., Macleod, I., Wilmot, A. J., and Elsdon-Dew, R. (1966). Lancet, 2, 1329.

Prata, A. (1963). Trans. roy. Soc. trop. Med. Hyg., 57, 266.

Rieselbach, R. E., Di Chiro, G., Freireich, E. J., and Rall, D. P. (1962). New Engl. J. Med., 267, 1273.

Sternberg, T. H., Wright, E. T., and Oura, M. (1956). Antibioticso Ann., 1955-56, 566. New York.

Taylor, R. L., Lynch, H., Taylor, R. R., and Weiser, O. L. (1958) Amer. Rev. Tuberc., 77, 1023.

Winn, W. A. (1963). Med. Clin. N. Amer., 47, 1131.

Witorsch, P., Williams, T. W. Jr, Ommaya, A. K., and Utz, J. P. (1965). J. Amer. med. Ass., 194, 699.

\section{ADDENDUM}

Since this paper was submitted for publication a Naegleria sp. has again been isolated from a case of the disease and shown by the cultural inhibition technique to have the same sensitivity to amphotericin B. Unfortunately, the patient (Adelaides Children's Hospital no. 69-1103) was in the terminatw stage of his illness and, as might be expected, dide not respond to treatment with this drug. 